\section{Light-sensitive Albino Tea Plants and Their Characterization}

\author{
Young-Hwan Shin, Rui Yang, Yun-Long Shi, Xu-Min Li, Qiu-Yue Fu, \\ Jian-Liang Lu, and Jian-Hui Ye \\ Tea Research Institute, Zhejiang University, Hangzhou 310058, China
}

\author{
Kai-Rong Wang \\ Ningbo Extension Station of Forestry \& Speciality Technology, Ningbo \\ 315012, China
}

Shi-Cheng Ma

Liupao Tea Academy, Wuzhou 543003, Guangxi, China

Xin-Qiang Zheng' ${ }^{1}$ and Yue-Rong Liang ${ }^{1}$
Tea Research Institute, Zhejiang University, Hangzhou 310058, China

Additional index words. Camellia sinensis, chemical composition, amino acids, carotenoids, chlorophylls, transcriptome

\begin{abstract}
Albino tea plants are mutants that grow albino young leaves owing to lack of chlorophylls under certain environmental conditions. There are two types of albino tea plants grown in production, i.e., light- and temperature-sensitive albino tea cultivars. The former grows albino leaves in yellow color under intensive sunlight conditions and the later grows albino leaves with white mesophyll and greenish vein as the environmental temperature is below $20^{\circ} \mathrm{C}$. Both albino teas attract great attention because of their high levels of amino acids and the "umami" taste. There have been many studies focusing on the temperature-sensitive albino tea plants, whereas little attention has been given to the light-sensitive albino tea cultivars. The characteristics of the albino tea cultivars and the mechanism underlying them were reviewed in the present article based on the published literatures, including chemical compositions, morphological characteristics, and molecular genetic mechanism.
\end{abstract}

There is great genetic variation in tea germplasm, among which albino tea plants are mutants that grow albino young leaves owing to lack of chlorophylls under certain environmental conditions (Du et al., 2006). The albino tea cultivars attract a great attention owing to their high level of amino acid concentration and especially the "umami" taste and fragrant aroma ( $\mathrm{Li}$ et al., 2016b). There are two types of albino tea plants found and used in production, i.e., temperaturesensitive and light-sensitive albino cultivars (Wang et al., 2015).

The temperature-sensitive albino tea cultivars grow "white" albino young leaves because of their reduced chlorophylls biosynthesis as environmental temperature is below 20 to $22{ }^{\circ} \mathrm{C}$ in the early Spring, but their chlorophyll biosynthesis resumes and the leaves become as green as common tea cultivars when the temperature rises above 20 to $22{ }^{\circ} \mathrm{C}$ in the summer and autumn seasons (Li et al., 1999). Light-sensitive albino tea cultivars grow young albino leaves under

Received for publication 18 Oct. 2017. Accepted for publication 29 Nov. 2017.

We acknowledge the Science Technology Department of Zhejiang Province for financial support to this work (Project No. 2016C02053-5).

${ }^{1}$ Corresponding authors. E-mail: xqzheng@zju. edu.cn or yrliang@zju.edu.cn. difference in total catechins between temperature-sensitive albino tea cultivar Baiye-1 (its former name was White leaf No. 1) and normal cultivar Fudingdabai although the former has higher level amino acids than the latter. Compared with temperature-sensitive albino tea cultivar Baiye-1, the light-sensitive albino tea cultivars have less total catechins although both amino acid levels are higher than normal tea cultivar Fudingdabai (Table 1) (Li et al., 2016d, 2016e).

Chlorophylls and carotenoids are important pigments in plants which play a key role in photosynthesis and coloration of leaves. Both light-sensitive and temperature-sensitive albino tea cultivars have much lower levels of chlorophylls $\mathrm{a}$ and $\mathrm{b}$ than normal tea cultivar such as Fudingdabai. Among the albino tea cultivars, the temperature-sensitive albino tea plants usually grow leaves with albino mesophyll and greenish leaf vein. That is why the temperature-sensitive albino tea plant has higher level of chlorophylls than the lightsensitive ones (Table 1). Carotenoids serve two key roles in plants, i.e., absorbing light energy for use in photosynthesis and protecting chlorophylls from photodamage (Armstrong and Hearst, 1996). Carotenoids are classified into two groups, i.e., the carotenes without oxygen such as $\beta$-carotene and the xanthophylls with oxygen such as neoxanthin, violaxanthin (V), and lutein. The light-sensitive albino cultivars have the highest level of $\mathrm{V}$ among the tested cultivars. However, the level of $\beta$-carotene in lightsensitive albino cultivars was much lower than those in normal cultivar Fudingdabai and temperature-sensitive albino tea cultivar Baiye-1. Carotenoids, including carotenes and xanthophylls, are a group of pigments in plant that protect photosynthetic organisms from damages induced by free radicals such as reactive oxygen species originating from water splitting, the first step of photosynthesis (Zakar et al., 2016). Also, carotenoids are indispensable for the function of the photosynthetic apparatus, and particularly in that of photosystem II (PS) because of their light harvesting and photoprotective capacity (Sozer et al., 2010; Stamatakis et al., 2014). Carotenoids can provide more efficient light absorption under low light conditions (Koyama et al., 1996). However, excessive energy needs to be reduced to avoid photoinhibition damage to the photosystems under high-light conditions (Powles, 1984). Carotenoids function to dissipate excessive energy or excitation nondestructively as heat, a phenomenon being called nonphotochemical quenching (NPQ) of chlorophyll a fluorescence (Leonelli et al., 2017). Plants with lightinduced zeaxanthin $(Z)$ establish larger NPQ and $\mathrm{Z}$ formation shapes the photosynthetic membrane into a responsive state against high-light exposure by enhancing the rate of NPQ formation (Ruban, 2016). The NPQ mechanism involves the conversion of $\mathrm{V}$ into $Z$. The interconversion of violaxanthin $(\mathrm{V})$, antheraxanthin (A), and zeaxanthin (Z) (VAZcycle) plays an important role in NPQ. During 
Table 1. Comparison of foliar chemical compositions between various tea cultivars. ${ }^{\mathrm{z}}$

\begin{tabular}{|c|c|c|c|c|c|c|}
\hline Cultivars & Yujinxiang ${ }^{y}$ & Huang- $2^{\mathrm{y}}$ & Huang- $8^{y}$ & Huang-13 & Baiye- $1^{x}$ & Fudingdabai ${ }^{\mathrm{w}}$ \\
\hline Total amino acids $\left(\mathrm{mg} \cdot \mathrm{g}^{-1}\right)$ & $56.85 \pm 1.09$ & $76.63 \pm 3.83$ & $73.37 \pm 1.67$ & $73.72 \pm 3.13$ & $64.52 \pm 1.48$ & $46.25 \pm 0.44$ \\
\hline Total catechins $\left(\mathrm{mg} \cdot \mathrm{g}^{-1}\right)$ & $175.83 \pm 1.07$ & $128.68 \pm 0.69$ & $141.31 \pm 1.58$ & $133.60 \pm 0.82$ & $196.80 \pm 6.83$ & $188.88 \pm 2.58$ \\
\hline Caffeine $\left(\mathrm{mg} \cdot \mathrm{g}^{-1}\right)$ & $39.71 \pm 0.25$ & $30.14 \pm 0.57$ & $28.18 \pm 0.22$ & $32.86 \pm 0.25$ & $28.40 \pm 0.78$ & $34.27 \pm 0.39$ \\
\hline Chlorophyll a $\left(\mu \mathrm{g} \cdot \mathrm{g}^{-1}\right)$ & $449.41 \pm 79.69$ & $206.60 \pm 64.16$ & $306.78 \pm 17.82$ & $311.45 \pm 21.87$ & $639.94 \pm 25.38$ & $1,325.05 \pm 308.29$ \\
\hline Chlorophyll b $\left(\mu \mathrm{g} \cdot \mathrm{g}^{-1}\right)$ & $134.51 \pm 11.32$ & $31.53 \pm 6.94$ & $76.06 \pm 25.26$ & $61.91 \pm 7.64$ & $282.04 \pm 16.80$ & $762.98 \pm 46.28$ \\
\hline$\beta$-carotene $\left(\mu \mathrm{g} \cdot \mathrm{g}^{-1}\right)$ & $158.72 \pm 12.19$ & $100.88 \pm 14.10$ & $112.99 \pm 6.31$ & $114.53 \pm 18.11$ & $146.20 \pm 7.35$ & $379.19 \pm 3.96$ \\
\hline Neoxanthin $\left(\mu g \cdot g^{-1}\right)$ & $78.09 \pm 8.22$ & $65.70 \pm 1.61$ & $65.86 \pm 3.06$ & $56.51 \pm 2.43$ & $49.92 \pm 0.56$ & $127.51 \pm 28.76$ \\
\hline Violaxanthin $\left(\mu g \cdot g^{-1}\right)$ & $45.40 \pm 6.71$ & $53.64 \pm 0.35$ & $52.09 \pm 4.27$ & $63.43 \pm 2.70$ & $26.72 \pm 0.00$ & $31.63 \pm 1.51$ \\
\hline Lutein $\left(\mu g \cdot g^{-1}\right)$ & $201.37 \pm 6.73$ & $174.70 \pm 0.55$ & $189.06 \pm 18.63$ & $221.31 \pm 1.21$ & $156.79 \pm 15.12$ & $373.77 \pm 22.27$ \\
\hline References & Li et al. (2016c) & Li et al. (2016b) & Li et al. (2016b) & Li et al. (2016b) & Li et al. (2016b) & Li et al. (2016c) \\
\hline
\end{tabular}

${ }^{\mathrm{z}}$ Shoots with two leaves and a bud were tested.

y Light-sensitive albino tea cultivars.

${ }^{\mathrm{x}}$ Temperature-sensitive albino tea cultivar and its former name was White leaf No. 1 .

${ }^{\mathrm{w}}$ Normal tea cultivar with green leaves.

exposure to excessive light, the enzyme violaxanthin deepoxidase (VDE) is activated and converts $\mathrm{V}$ to $\mathrm{Z}$ via the intermediate $\mathrm{A}$ (Yamamoto et al., 1999). In limiting light (or darkness), zeaxanthin epoxidase (ZEP) regenerates $\mathrm{V}$ through the addition of epoxy groups to the $\beta$-ionone rings of $\mathrm{Z}$ and $\mathrm{A}$ (Hieber et al., 2000). The high level of $V$ in light-sensitive cultivars (Table 1) might be interesting for their stress responses to high light. It was reported that a high light-inducible protein Synechocystis HliD binds chlorophyll a and $\beta$-carotene with a 3:1 ratio (Staleva et al., 2015). Table 1 showed that the ratio of chlorophyll a to $\beta$-carotene was around 3:1, with an exception of cultivars Yujinxiang and Baiye-1. $\beta$-carotene is essential for the assembly of PS II and PS I trimer, whereas xanthophylls including $\mathrm{V}$ can stabilize them (Zakar et al., 2016). Low level of $\beta$-carotene in the light-sensitive cultivars is considered to be related to their low level of chlorophyll a, and it might be an important factor leading to their light sensitivity.

The concentrations of neoxanthin and lutein in the light-sensitive albino tea cultivars lie between normal cultivar Fudingdabai and temperature-sensitive albino cultivar Baiye-1 (Table 1). Normally, the leaves of light-sensitive albino tea are in yellow color but those of temperature-sensitive albino tea are in white color (Fig. 1). The high levels of $\mathrm{V}$, lutein, and neoxanthin are considered to be responsible for the yellow color of the lightsensitive albino tea leaves after being unmasked by the disappearance of chlorophylls.

\section{Morphological Characteristics}

Table 2 shows the major trait characters of light-sensitive and temperature-sensitive albino cultivars. The leaves on the tender shoots of light-sensitive albino tea plants are always in yellow color owing to lack of chlorophylls and abundance of xanthophylls such as lutein, V, and neoxanthin. However, the mature leaves under the shield of canopy are in green color and they are the major contributors to the photosynthesis. The yellow leaf will become green after it is covered by a piece of paper for 1 week (Fig. 2) (Li et al., 2008), suggesting that the albino leaves in yellow color are induced by sunlight and
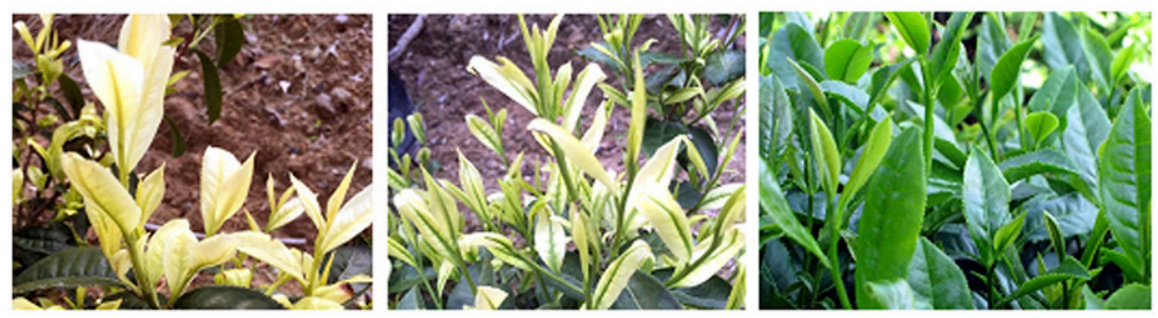

Fig. 1. Comparison of light-sensitive and temperature-sensitive albino tea leaves. Left: light-sensitive albino tea cultivar; Middle: temperature-sensitive albino tea cultivar; Right: normal cultivar.

the albino phenotype is reversible by sunshade. Because the leaves are sensitive to light, the young plants are easily injured in strong sunshine (Fig. 3A). The young plants with albino tender leaves but few mature green leaves are prone to be damaged by hot sunlight in the summer, resulting in low survival rate at the early stage after transplantation. In this case, the farmers are advised to shield their young tea plants using sunshade nets with $30 \%$ to $50 \%$ light transmittance in the first summer after transplanting (Fig. 3B) (Wang et al., 2008).

Hypoplasia of chloroplast development is found to be an important factor leading to the lack of chlorophylls and leaf albino phenotype. Under transmittance electronic microscopy, abnormal chloroplasts were observed in the light-induced albino leaves, showing less grana stacking and thylakoids. However, the grana stacking and thylakoids were enriched when the albino leaves were shield by sunshade nets. These suggest that intensive sunlight induces the hypoplasia of chloroplasts by suppressing the development of thylakoids and grana in the albino tea cultivar (Li et al., 2016b).

\section{Molecular Genetic Mechanism}

Molecular genetic studies show that the expression patterns of genes in light-sensitive albino tea plants involving in the metabolic pathways relating to chlorophylls and carotenoids are different from those in normal tea plants. Geranylgeranyl-PP is a product of 2C-methyl-D-erythritol-4-phosphate (MEP) pathway, and it is the precursor of carotenoids biosynthesized in carotenoid pathway and also the precursor of chlorophylls biosynthesized in chlorophyll pathway (Fig. 4). Compared with the normal tea cultivar Fudingdabai, the expression of the 1-deoxy-D-xylulose-5-phosphate synthases (DXSs, including DXS1, DXS2, and DXS3), a key enzyme in the MEP pathway, and also the gene magnesium chelatase subunit $\mathrm{D}$ (CHLD) in the chlorophyll pathway are downregulated by strong sunshine and upregulated by sunshade in the light-sensitive albino tea plants. However, the transcriptions of some genes involving in the carotenoid pathway such as phytoene synthase (PSY), phytoene desaturase (PDS), $\zeta$-carotene desaturase (ZDS), lycopene $\varepsilon$-cyclase (LCYE), lycopene $\beta$-cyclase (LCYB), carotene $\varepsilon$-monooxygenase (LUT1), ZEP, and VDE, are upregulated by strong sunshine in the light-sensitive albino tea plants (Li et al., 2016b). The expression pattern of the genes involving in the carotenoid pathway and chlorophyll pathway are consistent with the accumulations of chlorophylls and carotenoids in Table 1.

Digital gene expression profiling and quantitative real-time PCR (qRT-PCR) studies show that intensive light inhibits the expression of PS II $10-\mathrm{kDa}$ protein (PsbR) in light-sensitive albino tea cultivar Baijiguan, resulting in the reduction of chlorophyll biosynthesis and the less stability of PS II and chloroplast (Wu et al., 2016). The expressions of chloroplast genes PsbB, PsaB, PsaE, PsaF, PetA, and light-harvesting complex (LHC) chlorophyll $\mathrm{a} / \mathrm{b}$ binding protein 4 (Lhca 4) are downregulated in the lightsensitive albino tea cultivar Zhonghuang-1, compared with those in normal tea cultivar Longjing-43 (Wang et al., 2016). These genes are important for photosynthesis and growth of tea plants and their suppression by high light also explains why the survival rate 
Table 2. Major characters of light-sensitive and temperature-sensitive albino cultivars.

\begin{tabular}{|c|c|c|c|c|}
\hline \multirow[b]{2}{*}{ Cultivar name } & \multicolumn{2}{|c|}{ Light-sensitive albino tea } & \multicolumn{2}{|c|}{ Temperature-sensitive albino tea } \\
\hline & Baijiguan & Huangjinya & Xiaoxueya & Baiye- $1^{z}$ \\
\hline Species & Camellia sinensis & C. sinensis & C. sinensis & C. sinensis \\
\hline Propagation type & Asexual & Asexual & Asexual & Asexual \\
\hline Young shoot color & Yellow-green & Yellow & White & White with green vein \\
\hline Leaf shape & Oblong & Lanceolate & Elliptic & Oblong \\
\hline
\end{tabular}

${ }^{\mathrm{z}}$ Its former name was 'White leaf No. 1'.

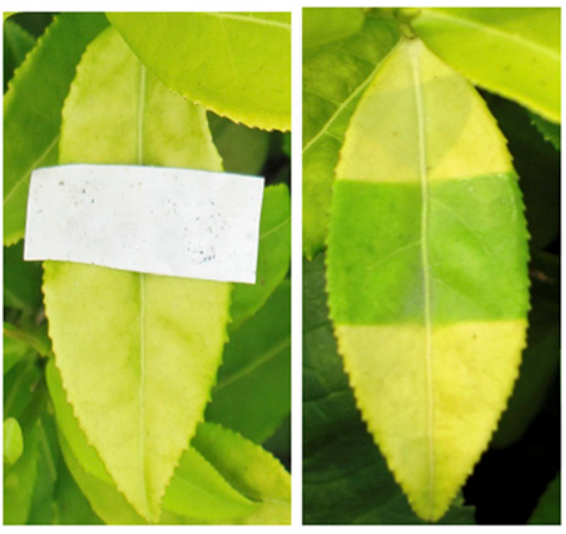

Fig. 2. Effect of sunshade on the leaf color of lightsensitive albino tea cultivar.
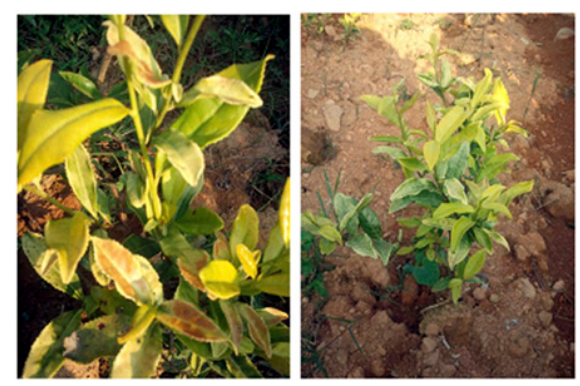

Fig. 3. Effect of sunlight shield on growth of albino tea cultivar. (A) Before sunlight shielding; (B) after sunlight shielding with $50 \%$ light transmittance sunshade net for 1 week.

of the transplanted young plants was low at the early stage after transplantation.

The expressions of genes involving in tea polyphenols biosynthesis in light-sensitive albino tea cultivars show different patterns from those in normal tea cultivars. Chalcone synthase (CHS) is the entry enzyme in flavonoid biosynthesis and it is a key enzyme in the catechins biosynthesis during the phenylpropanoid pathway (Mamati et al., 2006). Anthocyanidin reductase (ANR), an ANR, is the enzyme catalyzing the synthesis of anthocyanidin from flavan-3-ol in the presence of $\mathrm{NAD}^{+}$, and $\mathrm{NADP}^{+}$(Xie et al., 2003). Flavonol synthase (FLS) participates in flavonoid biosynthesis, in which it catalyzes the transformation of dihydroflavonol and 2-oxoglutarate into a flavonol and succinate in the presence of oxygen (Wellmann

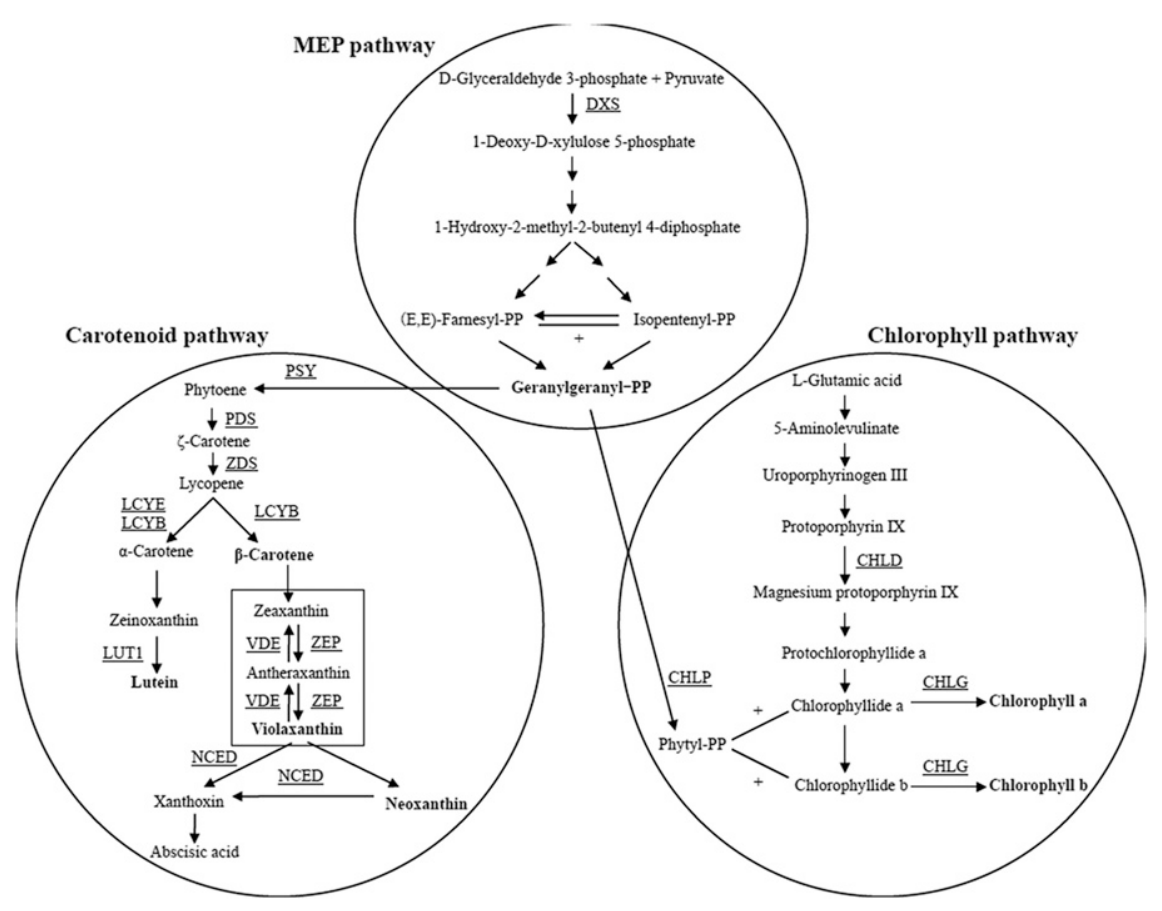

Fig. 4. Relationship of 2-C-methyl-D-erythritol-4-phosphate pathways to carotenoid pathway and chlorophyll pathway.

et al., 2002). In normal tea cultivars, these genes are upregulated under strong sunshine conditions in summer (Liang et al., 1996). However, the expressions of CHS, ANR, and FLS in the light-sensitive albino tea cultivar Zhonghuang-1 are suppressed by strong sunlight, compared with normal cultivar Longjing43 (Wang et al., 2016). This is the reason why the albino tea cultivars have less polyphenols than the normal tea cultivars.

Heat shock proteins (HSPs) are a family of proteins that are produced by cells in response to exposure to stressful conditions such as heat, cold, and ultraviolet light (Ritossa, 1962). Many members of HSPs perform chaperone function by stabilizing new proteins to ensure correct folding or by helping to refold proteins that were damaged by the cell stress (De Maio, 1999). The dramatic upregulation of the HSPs is a key part of the heat shock response and is induced primarily by a heat shock factor (Wu, 1995). Various HSPs were shown to be differentially expressed in the leaf and root of droughttolerant and drought-sensitive plants in response to drought. Upregulation of the HSPs is used to confer stress tolerance to hybridized plants, hoping to address drought and poor soil conditions for farming (Vinocur and Altman, 2005). Therefore, the downregulation of the HSPs HSP70 and HSP90 in cultivar Huangjinya under strong sunshine conditions is considered to be related to its light-sensitive albino properties (Song et al., 2017).

\section{Conclusion}

Both light-sensitive and temperaturesensitive albino tea cultivars contain high concentrations of amino acids and low levels of chlorophylls, and also the former contains less catechins than the latter. Because of expression upregulation of genes involving in carotenoid pathway including PSY, PDS, ZDS, LCYE, LCYB, LUT1, ZEP, and VDE, the light-sensitive albino tea cultivars contain higher levels of xanthophylls such as lutein, $\mathrm{V}$, and neoxanthin than the temperaturesensitive albino tea cultivar, resulting in yellow color of their leaves, whereas white color of the latter leaves. Hypoplasia of chloroplast development and expression downregulation of genes involving in chlorophylls biosynthesis are responsible for the 
albino leaf growth. In light-sensitive albino tea cultivars, the expressions of chloroplast genes PsbB, PsaB, PsaE, PsaF, PetA, LHC, and Lhca 4 are downregulated, resulting in the hypoplasia of chloroplast development, showing less grana stacking and thylakoids. The expression downregulation of genes involving in the MEP pathway, such as DXS, and genes involving in chlorophyll pathway, such as CHLD, are important factors leading to the lack of chlorophylls. The expression downregulation of genes involving in tea polyphenols biosynthesis, such as CHS, ANR, and FLS are leading factors for the reduction of catechins in the light-sensitive albino tea cultivars.

However, how the amino acids are highly accumulated in the light-sensitive albino tea leaves has not been understood so far. It is of great importance to clarify the mechanism of amino acid accumulation in the light-sensitive albino tea cultivars in the further studies.

\section{Literature Cited}

Armstrong, G.A. and J.E. Hearst. 1996. Carotenoids 2. Genetics and molecular biology of carotenoid pigment biosynthesis. FASEB J. 10:228-237.

De Maio, A. 1999. Heat shock proteins: Facts, thoughts, and dreams. Shock 11:1-12.

Du, Y.Y., H. Chen, W.L. Zhong, L.Y. Wu, J.H. Ye, C. Lin, X.Q. Zheng, J.L. Lu, and Y.R. Liang. 2008. Effect of temperature on accumulation of chlorophylls and leaf ultrastructure of low temperature induced albino tea plant. Afr. J. Biotechnol. 7:1881-1885.

Du, Y.Y., S. Shin, W.R. Wang, J.L. Lu, and Y.R. Liang. 2009. Effect of temperature on the expression of genes related to the accumulation of chlorophylls and carotenoids in albino tea. J. Hort. Sci. Biotechnol. 84:365-369.

Du, Y.Y., Y.R. Liang, H. Wang, K.R. Wang, J.L. Lu, G.H. Zhang, W.P. Lin, M. Li, and Q.Y. Fang. 2006. A study on the chemical composition of albino tea cultivars. J. Hortic. Sci. Biotechnol. 81:809-812.

Feng, L., M.J. Gao, R.Y. Hou, X.Y. Hu, L. Zhang, X.C. Wan, and S. Wei. 2014. Determination of quality constituents in the young leaves of albino tea cultivars. Food Chem. 155:98-104.

Hieber, A.D., R.C. Bugos, and H.Y. Yamamoto. 2000. Plant lipocalins: Violaxanthin deepoxidase and zeaxanthin epoxidase. Biochim. Biophys. Acta. 1482(1-2):84-91.

Koyama, Y., M. Kuki, P.O. Andersson, and T. Gillbro. 1996. Singlet excited states and the light-harvesting function of carotenoids in bacterial photosynthesis. Photochem. Photobiol. 63:243-256

Leonelli, L., M.D. Brooks, and K.K. Niyogi. 2017. Engineering the lutein epoxide cycle into Arabidopsis thaliana. Proc. Natl. Acad. Sci. USA 114:E7002-E7008.

Li, C.F., Y.X. Xu, J.Q. Ma, J.Q. Jin, D.J. Huang, M.Z. Yao, C.L. Ma, and L. Chen. 2016a. Biochemical and transcriptomic analyses reveal different metabolite biosynthesis profiles among three color and developmental stages in
'Anji Baicha' (Camellia sinensis). BMC Plant Biol. 16:195.

Li, M., L.J. Zhang, M. Shi, X.M. Lin, K.R. Wang, X.Q. Zheng, J.L. Lu, and Y.R. Liang. 2016b. Effects of light shading on chemical compositions of spring shoots in light-sensitive albino tea plants. Chaye 42:150-154. (in Chinese).

Li, M., L.J. Zhang, M. Shi, X.M. Lin, K.R. Wang, X.Q. Zheng, J.L. Lu, and Y.R. Liang. 2016c. Chemical composition of spring shoots in light-sensitive albino tea cultivar 'Yujinxiang'. Chaye 42:146-149. (in Chinese).

Li, M., L.J. Zhang, K.R. Wang, and Y.R. Liang. 2008. Studies on characteristics of illumination induced albino tea cultivar 'Huangjinya'. Chaye 34:98-102. (in Chinese).

Li, N.N., Y. Liu, Y. Zhao, X.Q. Zheng, J.L. Lu, and Y.R. Liang. 2016d. Simultaneous HPLC determination of amino acids in tea infusion coupled to pre-column derivatization with 2,4-dinitrofluorobenzene. Food Anal. Methods 9:1307-1314.

Li, N.N., Y.P. Yang, J.H. Ye, J.L. Lu, X.Q. Zheng, and Y.R. Liang. 2016e. Effects of sunlight on gene expression and chemical composition of light-sensitive albino tea plant. Plant Growth Regulat. 78:253-262.

Li, R.L. and Y.L. Kong. 2010. The research and development of albino tea cultivars. Jiangsu Agr. Sci. 6:12-15. (in Chinese).

Li, S.F., H. Cheng, M. Chen, F.L. Yu, J. Yan, Y.M. Liu, and L. Chen. 1999. Physiological and biochemical essence of the extraordinary characters of Anji Baicha. Chaye Kexue 19:87-92. (in Chinese).

Liang, Y.R., J.L. Lu, and S.L. Shang. 1996. Effect of gibberellins on chemical composition and quality of tea (Camellia sinensis L.). J. Sci. Food Agr. 72:411-414.

Liang, Y.R., Q. Ye, J. Jin, H. Liang, J.L. Lu, Y.Y. $\mathrm{Du}$, and J.J. Dong. 2008. Chemical and instrumental assessment of green tea sensory preference. Intl. J. Food Prop. 11:258-272.

Mamati, G.E., Y.R. Liang, and J.L. Lu. 2006. Expression of basic genes involved in tea polyphenol synthesis in relation to accumulation of catechins and total tea polyphenols. J. Sci. Food Agr. 86:459-464.

Powles, S.B. 1984. Photoinhibition of photosynthesis induced by visible-light. Annu. Rev. Plant Physiol. Plant Mol. Biol. 35:15-44.

Ritossa, F. 1962. A new puffing pattern induced by temperature shock and DNP in drosophila. Experientia 18:571-573.

Ruban, A.V. 2016. Non-photochemical chlorophyll fluorescence quenching: Mechanism and effectiveness in protection against photodamage. Plant Physiol. 170:1903-1916.

Song, L.B., Q.P. Ma, Z.W. Zou, K. Sun, Y.T. Yao, J.H. Tao, N.A. Kaeri, and X.H. Li. 2017. Molecular link between leaf coloration and gene expression of flavonoid and carotenoid biosynthesis in Camellia sinensis cultivar 'Huangjinya'. Front. Plant Sci. 8:803.

Sozer, O., J. Komenda, B. Ughy, I. Domonkos, H. Laczko-Dobos, P. Malec, Z. Gombos, and M. Kis. 2010. Involvement of carotenoids in the synthesis and assembly of protein subunits of photosynthetic reaction centers of Synechocystis sp. PCC6803. Plant Cell Physiol. 51:823-835.

Staleva, H., J. Komenda, M.K. Shukla, V. Slouf, R. Kana, T. Polivka, and R. Sobotka. 2015. Mechanism of photoprotection in the cyanobacterial ancestor of plant antenna proteins. Nat. Chem. Biol. 11:287-296.

Stamatakis, K., M. Tsimilli-Michael, and G.C. Papageorgiou. 2014. On the question of the light-harvesting role of beta-carotenein photosystem II and photosystem I core complexes. Plant Physiol. Biochem. 81:121-127.

Vinocur, B. and A. Altman. 2005. Recent advances in engineering plant tolerance to abiotic stress: Achievements and limitations. Curr. Opin. Biotechnol. 16:123-132.

Wang, K.R., M. Li, L.J. Zhang, and Y.R. Liang. 2015. Studies on classification of albino tea resources. Chaye 41:126-129. (in Chinese).

Wang, K.R., M. Li, Y.R. Liang, L.J. Zhang, L.M. Shen, and S.B. Wang. 2008. Breeding of new tea cultivar 'Huanginya'. China Tea 4:21-23. (In Chinese).

Wang, L., H.L. Cao, C.S. Chen, C. Yue, X.Y. Hao, Y.J. Yang, and X.C. Wang. 2016. Complementary transcriptomic and proteomic analyses of a chlorophyll-deficient tea plant cultivar reveal multiple metabolic pathway changes. J. Proteomics 130:160-169.

Wei, K., L.Y. Wang, J. Zhou, W. He, J.M. Zeng, Y.W. Jiang, and H. Cheng. 2012. Comparison of catechins and purine alkaloids in albino and normal green tea cultivars (Camellia sinensis L.) by HPLC. Food Chem. 130:720-724.

Wellmann, F., R. Lukacin, T. Moriguchi, L. Britsch, E. Schiltz, and U. Matern. 2002. Functional expression and mutational analysis of flavonol synthase from Citrus unshiu. Eur. J. Biochem. 269:4134-4142.

$\mathrm{Wu}, \mathrm{C} .1995$. Heat shock transcription factors: Structure and regulation. Annu. Rev. Cell Dev. Biol. 11:441-469.

Wu, Q.J., Z.D. Chen, W.J. Sun, T.T. Deng, and M.J. Chen. 2016. De novo sequencing of the leaf transcriptome reveals complex lightresponsive regulatory networks in Camellia sinensis cv. Baijiguan. Front. Plant Sci. 7:332.

Xie, D.Y., S.B. Sharma, N.L. Paiva, D. Ferreira, and R.A. Dixon. 2003. Role of anthocyanidin reductase, encoded by BANYULS in plant flavonoid biosynthesis. Science 299:396-399.

Xiong, L.G., J. Li, Y.H. Li, L. Yuan, S.Q. Liu, J.A. Huang, and Z.H. Liu. 2013. Dynamic changes in catechin levels and catechin biosynthesisrelated gene expression in albino tea plants (Camellia sinensis L.). Plant Physiol. Biochem. 71:132-143.

Xu, Y.X., C.J. Shen, J.Q. Ma, W. Chen, J. Mao, Y.Y. Zhou, and L. Chen. 2017. Quantitative succinyl-proteome profiling of Camellia sinensis cv. 'Anji Baicha' during periodic albinism. Sci. Rep. 7:1873.

Yamamoto, H.Y., R.C. Bugos, and A.D. Hieber. 1999Biochemistry and molecular biology of the xanthophyll cycle, p. 293-303. In: H.A. Frank, A.J. Young, G. Britton, and R.J. Cogdell (eds.). The photochemistry of carotenoids. Springer, Dordrecht, The Netherlands.

Yuan, L., L.G. Xiong, T.T. Deng, Y. Wu, J. Li, S.Q Liu, J.A. Huang, and Z.H. Liu. 2015. Comparative profiling of gene expression in Camellia sinensis L. cultivar Anji Baicha leaves during periodic albinism. Gene 561:23-29.

Zakar, T., H. Laczko-Dobos, T.N. Toth, and Z. Gombos. 2016. carotenoids assist in cyanobacterial photosystem II assembly and function. Front. Plant Sci. 7:295. 